\title{
Úlcera duodenal benigna con penetración en cabeza de páncreas, con obstrucción de la vía biliar
}

\author{
Javier Páramo Z. ${ }^{1}$, Laura Rubio L. ${ }^{1}$, Silvia Benito B. ${ }^{1}$, Ignacio Ortega F. ${ }^{1}$, \\ Jorge López ${ }^{2}$ y Arsenio Menchero S. ${ }^{1}$
}

\section{Benign duodenal ulcer penetrating into pancreas, with obstruction of common bile duct}

\begin{abstract}
Aim: To report an atypical presentation of a benign duodenal ulcer that simulates pancreatic neoplasia. Materials and Method: A case of a 83 years old male patient with astenia and jaundice due to a benign duodenal ulcer penetrating into the pancreas with obstruction of common bile duct. Imagining study identified a pancreatic head mass. The patient required one month admission, receiving broad-spectrum antibiotics, parenteral nutrition and intravenous proton pump inhibitors. Discussion and Conclusion: Due to frequent complications associated to this condition, such as haemodynamic failure, sepsis or free peritoneal perforation, surgery is the main treatment. However, in mild cases, as in our patient, conservative management can be considered. Penetration or fistulization to the head of the pancreas is a rare and serious complication of peptic ulcer disease. Its management can be conservative in selected cases where there is no perforation of the ulcer into the peritoneal cavity, nor septic or hemodynamic deterioration.
\end{abstract}

Key words: peptic ulcer; duodenal ulcer; pancreas; penetration; conservative therapy.

\section{Resumen}

Objetivo: Reportamos un caso clínico con presentación atípica de una úlcera duodenal benigna que simula el cuadro clínico y radiológico de una neoplasia de páncreas. Materiales y Método: Presentamos el caso de un varón de 83 años que debuta con un cuadro clínico de astenia e ictericia mucocutánea. En estudio de imagen se identifica una masa en cabeza pancreática. En estudio endoscópico se observa úlcera duodenal benigna penetrada a cabeza de páncreas que condiciona obstrucción de vía biliar. Discusión y Conclusiones: El manejo de estos pacientes suele ser quirúrgico porque desarrollan un deterioro asociado a sepsis o perforación. Si la situación clínica lo permite se puede intentar un tratamiento conservador. En nuestro caso el paciente precisó un mes de hospitalización con antibioticoterapia intravenosa de amplio espectro, reposo alimentario, nutrición parenteral y tratamiento con inhibidores de la bomba de protones (IBP) para la resolución del cuadro. La penetración o fistulización a la cabeza del páncreas es una complicación grave e infrecuente de la enfermedad ulcerosa péptica. Su manejo puede ser conservador en casos seleccionados donde no exista perforación de la úlcera a la cavidad peritoneal, ni exista deterioro séptico ni hemodinámico.

Palabras clave: úlcera péptica; úlcera duodenal; páncreas; penetración; terapia conservadora.

\section{Introducción}

Las complicaciones más frecuentes de la úlcera péptica (UP) de cualquier etiología son: El sangrado, la perforación, la penetración y la obstrucción ${ }^{1}$. Los casos de UP con penetración a la cabeza pancreática son extremadamente raros en la actualidad ${ }^{2}$. Presentamos el caso de un varón de 83 años con ictericia obstructiva y dolor abdominal secundarios a masa en cabeza pancreática, cavitada, y con gas en su interior, secundaria a úlcera duodenal complicada.

\section{Caso Clínico}

Varón de 83 años sin antecedentes médicos de interés ni clínica digestiva previa que acudió a ur-
'Servicio de Cirugía General y del Aparato Digestivo. ${ }^{2}$ Servicio de Aparato Digestivo. Hospital Universitario de Móstoles, Universidad Rey Juan Carlos.

Madrid, España.

Recibido 2020-05-20 y aceptado 2020-09-22

Correspondencia a: Dr. Javier Páramo Z. Javier.paramo@salud.madrid.org 


\section{CASOS CLÍNICOS}

gencias por dolor abdominal de 4 días de evolución asociado a ictericia y coluria. Reportó cuadro asténico y pérdida de peso de 2 meses de evolución. En la exploración física presentó dolor en el epigastrio, donde se palpó una masa intraabdominal. Ausencia de deterioro hemodinámico y fiebre.

En la analítica presentó leucocitosis de $16.540 \mathrm{c} / \mu \mathrm{L}$ (referencia 4.000-11.000 c/ $\mu \mathrm{L}$ ), neutrofilia de $90 \%$, amilasemia $131 \mathrm{U} / \mathrm{L}$ (referencia 0-137 U/L), Bilirrubina total $7,8 \mathrm{mg} / \mathrm{dL}$ (referencia 0,1 a $1,2 \mathrm{mg} / \mathrm{dL}$ ), Proteína C reactiva $287 \mathrm{mg} / \mathrm{L}$ (referencia 0-5 U/L) y CA $19.946 \mathrm{U} / \mathrm{mL}$ (referencia 0-37 U/mL).

En la ecografía urgente se observó una masa en cabeza pancreática sospechosa de malignidad que condicionaba dilatación de la vía biliar intra y extrahepática con un diámetro máximo de $13 \mathrm{~mm}$. No se identificó colecistitis asociada. El estudio se completó con una tomografía computarizada (TC) que identificó masa de 6 × 6 × $5 \mathrm{~cm}$ en la encrucija- da duodeno-biliopancreática con zona central hipodensa y burbujas de gas en su interior. Se observó afectación inflamatoria yuxtalesional de antro gástrico, duodeno, vía biliar y cabeza de páncreas; no pudiendo filiar la etiopatogenia de la lesión (Figura 1). Cortes axiales de TC donde se visualiza masa en cabeza de páncreas (Figura 2). Cortes coronales de TC donde se visualiza masa en cabeza de páncreas (Figura 3). Corte sagital de TC donde se visualiza la lesión pancreática. Se establecen inicialmente varias posibilidades diagnósticas: neoplasia de páncreas, colecistopancreatitis complicada o úlcera péptica complicada. El paciente es ingresado para soporte nutricional y antibioticoterapia intravenosa.

Se realiza estudio endoscópico donde se identificaron dos cavidades a nivel de la segunda porción duodenal con comunicación con la colección de la lesión pancreática. No se identificaron signos macroscópicos de malignidad. En el estudio anatomo-

Figura 1. Cortes axiales de TC donde se visualiza masa en cabeza de páncreas.

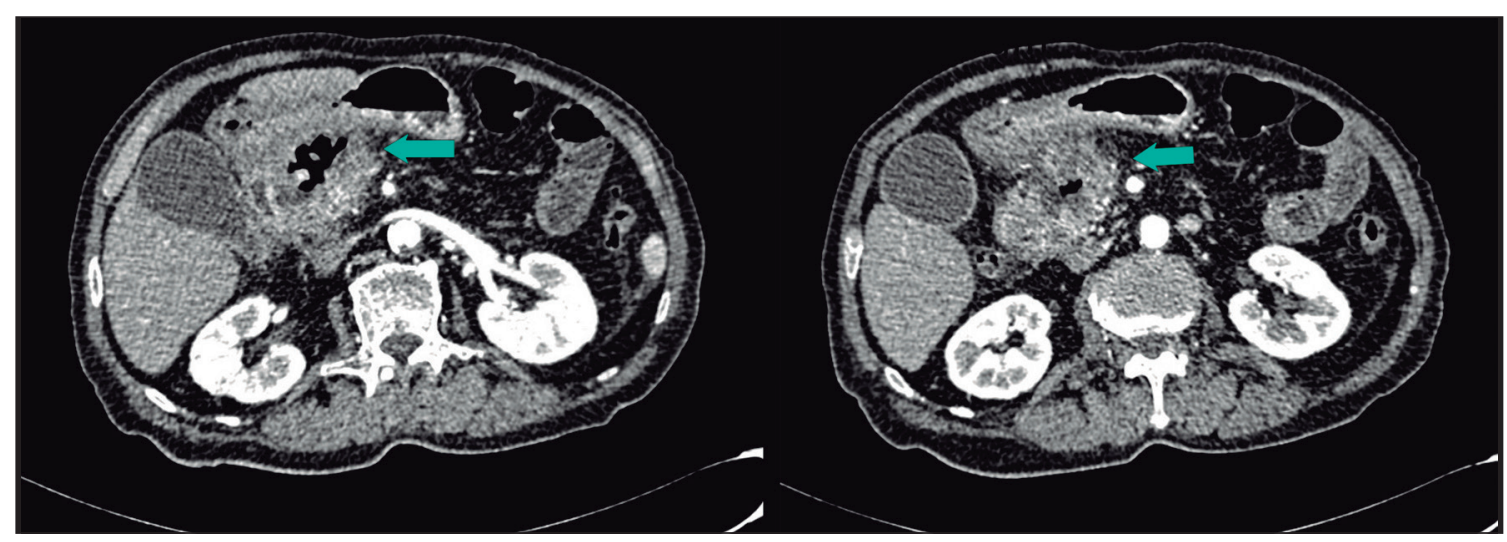

Figura 2. Cortes coronales de TC donde se visualiza masa en cabeza de páncreas.

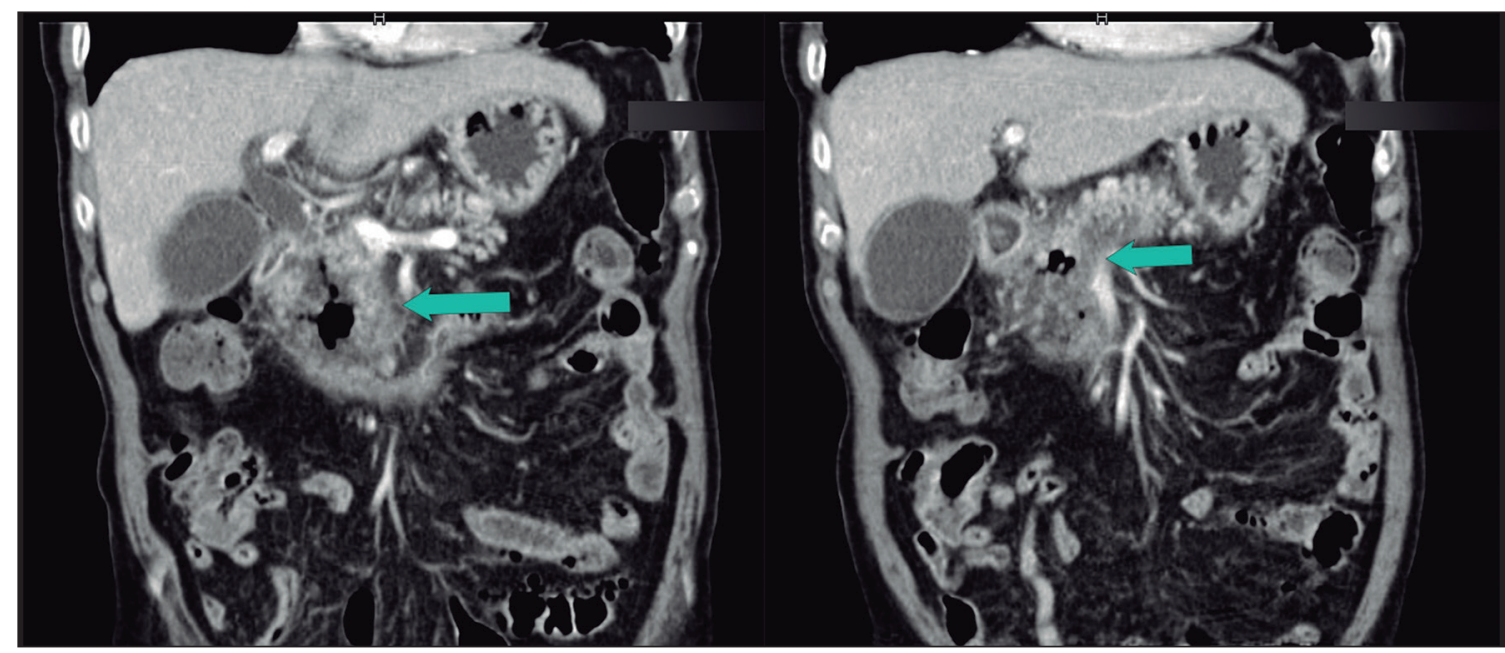


patológico de las biopsias endoscópicas se observó gastritis crónica antral con metaplasia y duodenitis aguda y linfoplasmocitaria con formación ocasional de folículos linfoides de centro germinal claro.

Dados los hallazgos radiológicos con ausencia de perforación libre en cavidad peritoneal, la avanzada edad del paciente y la evolución favorable en las primeras $48 \mathrm{~h}$ con tratamiento médico conservador, se decide mantener la actitud terapéutica y no indicar tratamiento quirúrgico. El tratamiento conservador consistió en reposo alimentario prolongado durante 15 días, soporte nutricional por vía parenteral durante 10 días, antibioticoterapia de amplio espectro (piperacilina-tazobactam, $4 \mathrm{~g}$ cada $6 \mathrm{~h}$, durante 15 días) y tratamiento intravenoso con inhibidor de bomba de protones (pantoprazol). El paciente tuvo una resolución total de la ictericia en la primera semana de ingreso y disminución del efecto masa en abdomen durante la exploración. Mejoría de la analítica a las 2 semanas del ingreso con una bilirrubina sérica total de $1,2 \mathrm{mg} / \mathrm{dL}$, una PCR de $19,30 \mathrm{mg} / \mathrm{L}$ y leucocitos $500 \mathrm{c} / \mu \mathrm{L}$. En TC previo al alta, y coincidiendo con el mes del ingreso, se observa práctica resolución de los hallazgos (Figura 4).

\section{Discusión}

La incidencia de la úlcera péptica (UP) en países occidentales ha disminuido drásticamente gracias a las mejoras en el conocimiento, manejo y tratamiento de la enfermedad ${ }^{1,3}$. No obstante, el riesgo de complicaciones graves prácticamente no se ha modificado y se estima una incidencia en torno a $1 \%-2 \%$ anual por paciente con diagnóstico de úlcera $^{4,5}$. Las complicaciones pueden aparecer en la
UP de cualquier etiología, siendo las más frecuentes: la hemorragia, la perforación, la penetración y la obstrucción gástrica ${ }^{6}$. En nuestro paciente la enfermedad ulcerosa debuta con una masa de naturaleza, inicialmente, indeterminada en la cabeza pancreática, secundaria a penetración tisular de la lesión, que condiciona dilatación de la vía biliar intra y extrahepática de hasta $15 \mathrm{~mm}$ e ictericia. Simula clínica y radiológicamente una neoplasia avanzada de la cabeza del páncreas. La penetración es patogénicamente similar a la perforación, aunque en la primera la úlcera erosiona y excava el tejido de algún órgano vecino (como el hígado o páncreas) y

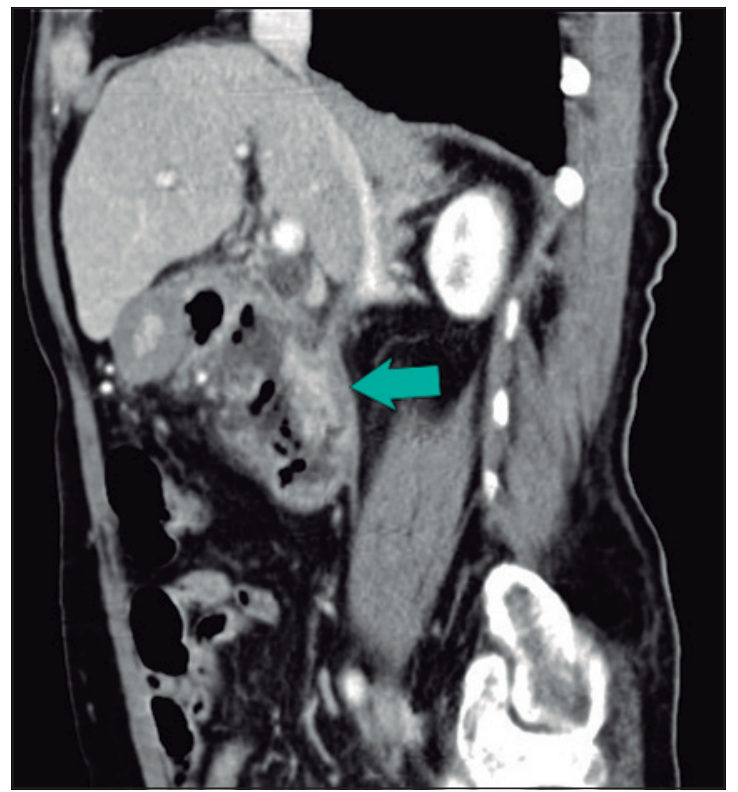

Figura 3. Corte sagital de TC donde se visualiza masa en cabeza de páncreas.

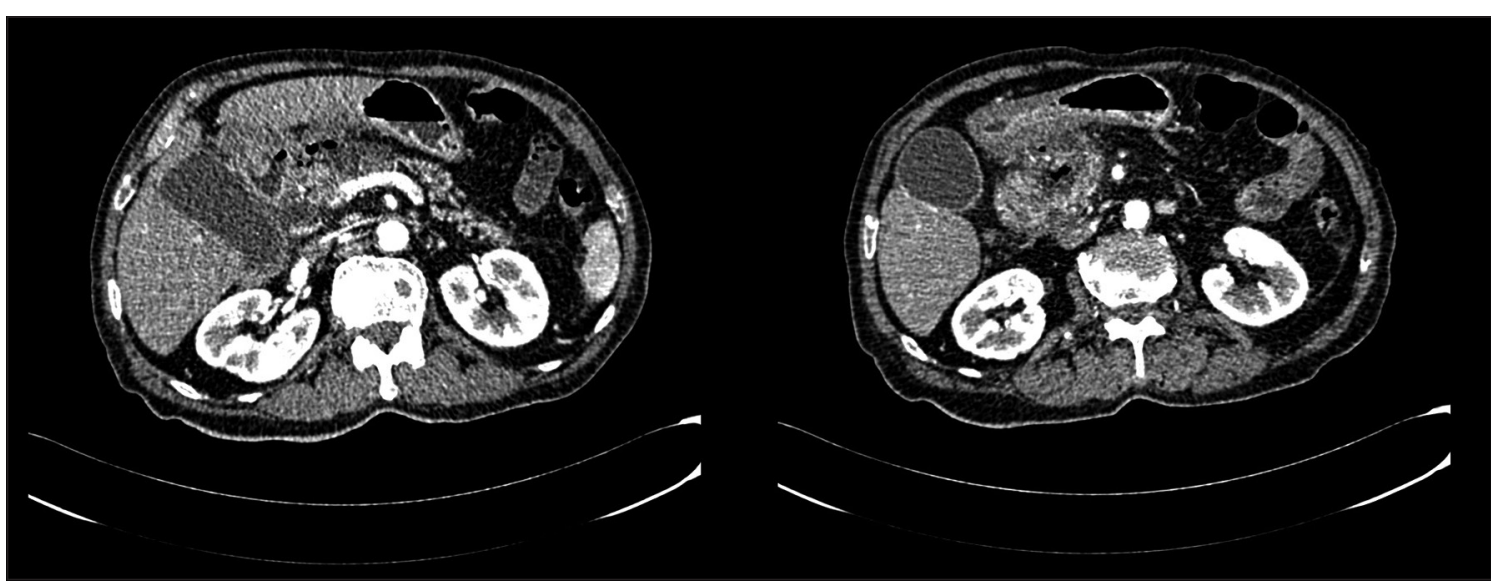

Figura 4. Cortes axiales de TC al mes del ingreso en planta, donde se observa notable mejoría de la lesión. 
no se abre a cavidad peritoneal ${ }^{7}$. Existen pocos casos reportados en base de datos PubMed de enfermedad ulcerosa penetrada a páncreas ${ }^{2,8,9}$. El manejo de estos pacientes suele ser quirúrgico porque desarrollan un deterioro hemodinámico asociado a sepsis o perforación peritoneal. No obstante, si la situación clínica del paciente lo permite, como sucede en nuestro caso, se puede optar por un tratamiento conservador $^{2}$. En nuestro caso, el paciente precisó un mes de hospitalización con antibioticoterapia intravenosa de amplio espectro, reposo alimentario, nutrición parenteral y tratamiento con IBP para la resolución del cuadro.

\section{Conclusión}

La penetración o fistulización a la cabeza del páncreas es una complicación grave e infrecuente de la enfermedad ulcerosa péptica. Su diagnóstico inicial puede ser difícil al simular otras entidades, como la tumoración maligna de cabeza de páncreas. Es preciso el uso de diferentes técnicas diagnósticas (ecografía, TC, resonancia magnética, endoscopia) para el diagnóstico diferencial. Su manejo puede ser conservador en casos seleccionados donde no exista perforación de la úlcera a la cavidad peritoneal, no exista deterioro hemodinámico, o la avanzada edad del paciente no permita tratamiento quirúrgico agresivo.

\section{Agradecimientos}

Gracias a mis compañeros del Hospital Universitario de Móstoles por ofrecer siempre calidad y humanidad en su atención a los pacientes.

\section{Responsabilidades éticas}

Protección de personas y animales. Los autores declaran que para esta investigación no se han realizado experimentos en seres humanos ni en animales.

Confidencialidad de los datos. Los autores declaran que en este artículo no aparecen datos de pacientes.

Conflictos de interés: no hay.

Financiación: Se declara que los autores no han recibido ni recibirán remuneración alguna por este artículo. Todo fue realizado con recursos propios de los autores.

\section{Bibliografía}

1. Post PN, Kuipers EJ, Meijer GA. Declining incidence of peptic ulcer but not of its complications: a nation-wide study in The Netherlands. Aliment Pharmacol Ther. 2006;23:1587-93.

2. Shibukawa N, Ouchi S, Wakamatsu S, Wakahara Y, Tatsumi N, Kaneko A. A Rare Case of Gastric Ulcer Penetrating the Pancreas that was Successfully Managed by Conservative Therapy. Intern Med. 2017;56:2277-9.

3. Gustavsson S, Kelly KA, Melton LJ,
Zinsmeister AR. Trends in peptic ulcer surgery. A population-based study in Rochester, Minnesota, 1956-1985. Gastroenterology 1988;94:688-94.

4. Irazola AC, García JLC, Arbeloa AL Complicaciones de la úlcera péptica. Med Programa Form Médica Contin Acreditado. 2008;10:141-7.

5. Behrman SW. Management of complicated peptic ulcer disease. Arch Surg Chic Ill 1960. 2005;140: 201-8.

6. Milosavljevic T, Kostić-Milosavljević M,
Jovanović I, Krstić M. Complications of peptic ulcer disease. Dig Dis Basel Switz. 2011;29:491-3.

7. Lowel J. Peptic ulcer. In: Rosen's Emergency Medicine. 8th ed. Philadelphia: Elsevier; 2013.

8. Fujihara S, Mori H, Nishiyama N, Kobara H, Masaki T. Giant gastric ulcer penetrating into the pancreas. Arab J Gastroenterol Off Publ Pan-Arab Assoc Gastroenterol. 2012;13:158-60.

9. Volwiler W, Mallory TB. Benign gastric ulcer, with penetration into pancreas. $\mathrm{N}$ Engl J Med. 1946;235:201. 